\title{
Existence results to certain functional equations in probabilistic Banach spaces with an application to integral equations
}

\author{
Mohamed Jleli, Bessem Samet* \\ Department of Mathematics, College of Science, King Saud University, P.O. Box 2455, Riyadh 11451, Saudi Arabia. \\ Communicated by Z. Kadelburg
}

\begin{abstract}
We consider some classes of functional equations posed in PB-spaces, for which we establish existence and uniqueness of solutions that belong to a cone. An application to integral equations is presented. (C)2016 All rights reserved.
\end{abstract}

Keywords: Functional equation, PB-space, normal cone, partial order, integral equation. 2010 MSC: 47H07, 47H05, 45G10.

\section{Introduction}

Many problems in physics can be described via operator equations posed in a certain space. It is very important to know if such equations admit solutions and if a solution exists, it is unique. Many authors studied such questions and considered various classes of operator equations posed in a Banach space (see, for examples, 11, 2, 3, 7, 9, 12, 13, 14, 15, 16]). In [8, we studied a class of operator equations posed in a probabilistic Banach space and involving decreasing and convex operators. In this contribution, we continue our study for other classes of functional equations. Let us start by recalling some basic concepts and results about probabilistic Banach spaces that will be used through this paper. For more details, the reader is invited to consult [3, 4, 5, 6, 7, 8, 10, 11].

We denote by $\mathbb{D}$ the set of distribution mappings. A probabilistic norm on a $\mathbb{R}$-vector space $\mathbb{V}$ w.r.t a $T$-norm $\mathbb{T}$ is a mapping $N: \mathbb{V} \rightarrow \mathbb{D}$ having the following properties:

\footnotetext{
* Corresponding author

Email addresses: jleli@ksu.edu.sa (Mohamed Jleli), bsamet@ksu.edu.sa (Bessem Samet)
} 
$(\mathrm{N} 1) N(v)(0)=0$ for every $v \in \mathbb{V}$;

(N2) $N(v)(\lambda)=1$ for all $\lambda>0$ iff $v=0$;

(N3) $N(\delta v)(\lambda)=N(v)\left(\frac{\lambda}{|\delta|}\right)$ for all $v \in \mathbb{V}$ and $\delta \in \mathbb{R}, \delta \neq 0$;

(N4) $N(u+v)(\lambda+\mu) \geq \mathbb{T}(N(u)(\lambda), N(v)(\mu))$ for all $u, v \in \mathbb{V}$ and $\lambda, \mu \geq 0$.

In this case, $(\mathbb{V}, N, \mathbb{T})$ is a probabilistic normed space (shortly $P N$-space).

For topological concepts in $P N$-spaces, see [8]. A probabilistic Banach space (shortly $P B$-space) is a $P N$-space, for which every Cauchy sequence is convergent.

Let $(\mathbb{V}, N, \mathbb{T})$ be a $P B$-space and $Q$ be a convex subset of $\mathbb{V}$ such that $Q \neq \emptyset$. Under the conditions: (Q1) $\bar{Q}=Q$;

(Q2) $q Q \subseteq Q$ for every $q \geq 0$;

(Q3) $-Q \cap Q=\{0\}$,

we say that $Q$ is a cone in $P B$. In this case, the binary relation $\preceq$ defined by

$$
z, w \in \mathbb{V}, \quad z \preceq w \Longleftrightarrow w-z \in Q
$$

is a partial order in $\mathbb{V}$. For a pair $(u, v)$ that belongs to $\mathbb{V} \times \mathbb{V}$, we use the notation $u \prec v$ to indicate that $v-u \in Q$ and $u \neq v$. In this case, the interval $[u, v]$ is the set of elements $z \in \mathbb{V}$ such that $z-u \in Q$ and $v-z \in Q$. For a given $\eta \in Q$, we denote by $Q_{\eta}$ the set of elements $v \in \mathbb{V}$ such that $v-\lambda \eta \in Q$ and $\mu \eta-v \in Q$ for some $\lambda, \mu>0$. The cone $Q$ is normal if there is some constant $\rho>0$ such that

$$
(u, v) \in \mathbb{V} \times \mathbb{V}, 0 \preceq u \preceq v \Longrightarrow N(u)(x) \geq N(v)\left(\frac{x}{\rho}\right), x \in \mathbb{R} .
$$

Now, we are able to present and establish our obtained results.

\section{Main result and consequences}

In the sequel, $(\mathbb{V}, N, \mathbb{T})$ denotes a PB-space with a normal cone $Q$. Let $\zeta:(\alpha, \beta) \rightarrow(0,1)$ be a surjective function and $\xi:(\alpha, \beta) \times Q \times Q \rightarrow(0, \infty)$ be a function having the following properties:

$\left(\xi_{1}\right) \xi(x, v, w) \in(\zeta(x), 1)$ for all $x \in(\alpha, \beta),(v, w) \in Q \times Q$;

$\left(\xi_{2}\right)$ for every $x \in(\alpha, \beta), \xi(x, v, w)$ is increasing in $v$ (w.r.t $\preceq$ ) for $w$ fixed and decreasing in $w$ for $v$ fixed.

We denote by $\mathcal{F}$ the set of operators $F: Q \times Q \rightarrow Q$ such that

(F1) $F$ is mixed monotone;

(F2) for all $(x, v, w) \in(\alpha, \beta) \times Q \times Q$, we have

$$
F\left(\zeta(x) v,[\zeta(x)]^{-1} w\right) \succeq \xi(x, v, w) F(v, w) .
$$

We denote by $\mathcal{G}$ the set of operators $G: \mathbb{V} \rightarrow \mathbb{V}$ such that

$\left(\mathcal{G}_{1}\right) G$ is increasing;

$\left(\mathcal{G}_{2}\right)$ for all $(p, v) \in(0,1) \times Q$, we have

$$
G(p v) \succeq p G v .
$$

Our main result in this paper is the following.

Theorem 2.1. Let $(F, G) \in \mathcal{F} \times \mathcal{G}$. Suppose that there exists $\eta \in Q, \eta \neq 0$ such that

$$
\frac{\zeta\left(x_{0}\right)}{\xi\left(x_{0}, \eta, \eta\right)} \eta \preceq G F(\eta, \eta) \preceq \frac{1}{\zeta\left(x_{0}\right)} \eta,
$$

for some $x_{0} \in(\alpha, \beta)$. Then the operator equation

$$
G F(v, v)=v
$$

has a unique solution $v^{*} \in Q_{\eta}$. 
Proof. Define the operator $H: Q \times Q \rightarrow \mathbb{V}$ by

$$
H(v, w)=G F(v, w), \quad(v, w) \in Q \times Q .
$$

Observe that from $\left(\mathcal{G}_{2}\right)$, we have

$$
G 0 \succeq \frac{1}{2} G 0,
$$

which yields

$$
G 0 \in Q .
$$

Sine $G$ is increasing, we get $H(Q \times Q) \subseteq Q$. Then $H: Q \times Q \rightarrow Q$. Let $\left(\phi_{1}, \omega_{1}\right),\left(\phi_{2}, \omega_{2}\right) \in Q \times Q$ with $\phi_{2}-\phi_{1} \in Q$ and $\omega_{1}-\omega_{2} \in Q$. From (F1), we have

$$
F\left(\phi_{1}, \omega_{1}\right) \preceq F\left(\phi_{2}, \omega_{2}\right) .
$$

Then from $\left(\mathcal{G}_{1}\right)$, we get

$$
G F\left(\phi_{1}, \omega_{1}\right) \preceq G F\left(\phi_{2}, \omega_{2}\right),
$$

that is,

$$
H\left(\phi_{1}, \omega_{1}\right) \preceq H\left(\phi_{2}, \omega_{2}\right) .
$$

This proves that $H$ is mixed monotone. Using $(\mathrm{F} 2),\left(\mathcal{G}_{1}\right)$ and $\left(\mathcal{G}_{2}\right)$, for all $(x, \phi, \omega) \in(\alpha, \beta) \times Q \times Q$, we obtain

$$
\begin{aligned}
H\left(\zeta(x) \phi,[\zeta(x)]^{-1} \omega\right) & =G F\left(\zeta(x) \phi,[\zeta(x)]^{-1} \omega\right) \\
& \succeq G(\xi(x, \phi, \omega) F(\phi, \omega)) \\
& \succeq \xi(x, \phi, \omega) G F(\phi, \omega) \\
& =\xi(x, \phi, \omega) H(\phi, \omega),
\end{aligned}
$$

that is,

$$
H\left(\zeta(x) \phi,[\zeta(x)]^{-1} \omega\right) \succeq \xi(x, \phi, \omega) H(\phi, \omega), \quad(x, \phi, \omega) \in(\alpha, \beta) \times Q \times Q .
$$

Since $\xi\left(x_{0}, \eta, \eta\right) \in\left(\zeta\left(x_{0}\right), 1\right)$, there is some positive integer $m$ such that

$$
\left(\frac{\xi\left(x_{0}, \eta, \eta\right)}{\zeta\left(x_{0}\right)}\right)^{m}>\left[\zeta\left(x_{0}\right)\right]^{-1}
$$

Set

$$
\phi_{0}=\left[\zeta\left(x_{0}\right)\right]^{m} \eta \quad \text { and } \quad \omega_{0}=\left[\zeta\left(x_{0}\right)\right]^{-m} \eta \text {. }
$$

Observe that

$$
\phi_{0}, \omega_{0} \in Q_{\eta} \quad \text { and } \quad \phi_{0}=\left[\zeta\left(x_{0}\right)\right]^{2 m} \omega_{0} \prec \omega_{0} .
$$

Moreover, take $0<\varepsilon \leq\left[\zeta\left(x_{0}\right)\right]^{2 m}$, we obtain

$$
\phi_{0} \succeq \varepsilon \omega_{0} .
$$

Using $\left(\xi_{1}\right),(2.1)$ and (2.3), we obtain

$$
\begin{aligned}
H\left(\phi_{0}, \omega_{0}\right) & =H\left(\left[\zeta\left(x_{0}\right)\right]^{m} \eta,\left[\zeta\left(x_{0}\right)\right]^{-m} \eta\right) \\
& =H\left(\left[\zeta\left(x_{0}\right)\right]\left[\zeta\left(x_{0}\right)\right]^{m-1} \eta,\left[\zeta\left(x_{0}\right)\right]^{-1}\left[\zeta\left(x_{0}\right)\right]^{1-m} \eta\right) \\
& \succeq \xi\left(x_{0},\left[\zeta\left(x_{0}\right)\right]^{m-1} \eta,\left[\zeta\left(x_{0}\right)\right]^{1-m} \eta\right) H\left(\left[\zeta\left(x_{0}\right)\right]^{m-1} \eta,\left[\zeta\left(x_{0}\right)\right]^{1-m} \eta\right) \\
& \succeq \xi\left(x_{0},\left[\zeta\left(x_{0}\right)\right]^{m-1} \eta,\left[\zeta\left(x_{0}\right)\right]^{1-m} \eta\right) \xi\left(x_{0},\left[\zeta\left(x_{0}\right)\right]^{m-2} \eta,\left[\zeta\left(x_{0}\right)\right]^{2-m} \eta\right) H\left(\left[\zeta\left(x_{0}\right)\right]^{m-2} \eta,\left[\zeta\left(x_{0}\right)\right]^{2-m} \eta\right) \\
& \vdots \\
& \succeq \xi\left(x_{0},\left[\zeta\left(x_{0}\right)\right]^{m-1} \eta,\left[\zeta\left(x_{0}\right)\right]^{1-m} \eta\right) \cdots \xi\left(x_{0}, \eta, \eta\right) H(\eta, \eta) \\
& \succeq\left[\zeta\left(x_{0}\right)\right]^{m-1} \xi\left(x_{0}, \eta, \eta\right) H(\eta, \eta) \\
& \succeq\left[\zeta\left(x_{0}\right)\right]^{m} \eta=\phi_{0} .
\end{aligned}
$$


On the other hand, from 2.3 , we can write

$$
H\left([\zeta(x)]^{-1} \phi, \zeta(x) \omega\right) \preceq \frac{1}{\xi\left(x,[\zeta(x)]^{-1} \phi, \zeta(x) \omega\right)} H(\phi, \omega), \quad(x, \phi, \omega) \in(\alpha, \beta) \times Q \times Q .
$$

Using $\left(\xi_{2}\right), 2.1$, 2.4 and (2.5), we get

$$
\begin{aligned}
H\left(\phi_{0}, \omega_{0}\right) & =H\left(\left[\zeta\left(x_{0}\right)\right]^{-m} \eta,\left[\zeta\left(x_{0}\right)\right]^{m} \eta\right) \\
& =H\left(\left[\zeta\left(x_{0}\right)\right]^{-1}\left[\zeta\left(x_{0}\right)\right]^{1-m} \eta,\left[\zeta\left(x_{0}\right)\right]\left[\zeta\left(x_{0}\right)\right]^{m-1} \eta\right) \\
& \preceq \frac{1}{\xi\left(x_{0},\left[\zeta\left(x_{0}\right)\right]^{-m} \eta,\left[\zeta\left(x_{0}\right)\right]^{m} \eta\right)} H\left(\left[\zeta\left(x_{0}\right)\right]^{1-m} \eta,\left[\zeta\left(x_{0}\right)\right]^{m-1} \eta\right) \\
& \preceq \frac{1}{\xi\left(x_{0},\left[\zeta\left(x_{0}\right)\right]^{-m} \eta,\left[\zeta\left(x_{0}\right)\right]^{m} \eta\right)} \frac{1}{\xi\left(x_{0},\left[\zeta\left(x_{0}\right)\right]^{1-m} \eta,\left[\zeta\left(x_{0}\right)\right]^{m-1} \eta\right)} H\left(\left[\zeta\left(x_{0}\right)\right]^{2-m} \eta,\left[\zeta\left(x_{0}\right)\right]^{m-2} \eta\right) \\
& \vdots \\
& \preceq \frac{1}{\xi\left(x_{0},\left[\zeta\left(x_{0}\right)\right]^{-m} \eta,\left[\zeta\left(x_{0}\right)\right]^{m} \eta\right)} \cdots \frac{1}{\xi\left(x_{0},\left[\zeta\left(x_{0}\right)\right]^{-1} \eta,\left[\zeta\left(x_{0}\right)\left(x_{0}\right)\right] \eta\right)} H(\eta, \eta) \\
& \preceq\left(\frac{1}{\xi\left(x_{0}, \eta, \eta\right)}\right)^{m} H(\eta, \eta) \\
& \preceq\left(\frac{1}{\xi\left(x_{0}, \eta, \eta\right)}\right)^{m} \frac{1}{\zeta\left(x_{0}\right)} \eta \\
& =\left[\zeta\left(x_{0}\right)\right]^{-m} \eta=\omega_{0} .
\end{aligned}
$$

As consequence, we have

$$
\phi_{0} \preceq H\left(\phi_{0}, \omega_{0}\right) \preceq H\left(\omega_{0}, \phi_{0}\right) \preceq \omega_{0} .
$$

Let

$$
\phi_{n+1}=H\left(\phi_{n}, \omega_{n}\right), \omega_{n+1}=H\left(\omega_{n}, \phi_{n}\right), \quad n=0,1,2, \cdots
$$

Then we have

$$
\phi_{0} \preceq \phi_{1} \preceq \omega_{1} \preceq \omega_{0} .
$$

By induction, we obtain easily

$$
\phi_{0} \preceq \phi_{1} \preceq \cdots \preceq \phi_{n} \preceq \cdots \preceq \omega_{n} \preceq \cdots \preceq \omega_{1} \preceq \omega_{0} .
$$

Set

$$
s_{n}=\sup \left\{s>0: \phi_{n} \succeq s \omega_{n}\right\}, \quad n=0,1,2, \cdots
$$

Then we have

$$
\phi_{n} \succeq s_{n} \omega_{n}, \quad n=0,1,2, \cdots,
$$

which implies from $(2.6)$ that

$$
\phi_{n+1} \succeq \phi_{n} \succeq s_{n} \omega_{n} \succeq s_{n} \omega_{n+1}, \quad n=0,1,2, \cdots,
$$

which yields

$$
0<s_{0} \leq s_{1} \leq \cdots \leq s_{n} \leq s_{n+1} \leq \cdots \leq 1 .
$$

Then there exists some $s \in(0,1]$ such that

$$
\lim _{n \rightarrow \infty} s_{n}=s .
$$

Suppose $s \in(0,1)$. We distinguish two cases.

Case 1. $s_{N}=s$ for some positive integer $N$.

In this case, we get

$$
s_{n}=s, \quad n \geq N,
$$


which yields

$$
\phi_{n} \succeq s \omega_{n}, \quad n \geq N .
$$

Since $s \in(0,1)$, there is some $x_{s} \in(\alpha, \beta)$ such that $\zeta\left(x_{s}\right)=s$. Using $(2.3),\left(\xi_{1}\right)$ and (2.6), for all $n \geq N$, we get

$$
\begin{aligned}
\phi_{n+1} & =H\left(\phi_{n}, \omega_{n}\right) \\
& \succeq H\left(s \omega_{n}, s^{-1} \phi_{n}\right) \\
& =H\left(\zeta\left(x_{s}\right) \omega_{n},\left[\zeta\left(x_{s}\right)\right]^{-1} \phi_{n}\right) \\
& \succeq \xi\left(x_{s}, \omega_{n}, \phi_{n}\right) H\left(\omega_{n}, \phi_{n}\right) \\
& \succeq \xi\left(x_{s}, \phi_{0}, \omega_{0}\right) \omega_{n+1} .
\end{aligned}
$$

The above inequality with $\left(\xi_{1}\right)$ yield

$$
s=\alpha\left(x_{s}\right)<\xi\left(t x_{s}, \phi_{0}, \omega_{0}\right) \leq s_{n+1}=s, \quad n \geq N,
$$

which is a contradiction.

Case 2. $s_{n}<s$ for every $n$.

In this case, we have

$$
0<\frac{s_{n}}{s}<1, \quad n=1,2,3, \cdots
$$

Then

$$
\frac{s_{n}}{s}=\zeta\left(q_{n}\right), \quad n=1,2,3, \cdots
$$

for some $q_{n} \in(\alpha, \beta)$. Then

$$
\begin{aligned}
\phi_{n+1} & =H\left(\phi_{n}, \omega_{n}\right) \\
& \succeq H\left(s_{n} \omega_{n}, s_{n}^{-1} \phi_{n}\right) \\
& =H\left(\zeta\left(q_{n}\right) s \omega_{n},\left[\zeta\left(q_{n}\right)\right]^{-1} s^{-1} \phi_{n}\right) \\
& \succeq \xi\left(q_{n}, s \omega_{n}, s^{-1} \phi_{n}\right) A\left(s \omega_{n}, s^{-1} \phi_{n}\right) \\
& \succeq \xi\left(q_{n}, s \phi_{0}, s^{-1} \omega_{0}\right) A\left(s \omega_{n}, s^{-1} \phi_{n}\right) \\
& \succeq \xi\left(q_{n}, s \phi_{0}, s^{-1} \omega_{0}\right) H\left(\zeta\left(x_{s}\right) \omega_{n},\left[\zeta\left(x_{s}\right)\right]^{-1} \phi_{n}\right) \\
& \succeq \xi\left(q_{n}, s \phi_{0}, s^{-1} \omega_{0}\right) \xi\left(x_{s}, \omega_{n}, \phi_{n}\right) H\left(\omega_{n}, \phi_{n}\right) \\
& \succeq \xi\left(q_{n}, s \phi_{0}, s^{-1} \omega_{0}\right) \xi\left(x_{s}, \phi_{0}, \omega_{0}\right) \omega_{n+1} .
\end{aligned}
$$

This yields

$$
\frac{s_{n}}{s} \xi\left(x_{s}, \phi_{0}, \omega_{0}\right)<\xi\left(q_{n}, s \phi_{0}, s^{-1} \omega_{0}\right) \xi\left(x_{s}, \phi_{0}, \omega_{0}\right) \leq s_{n+1} .
$$

Passing to the limit as $n \rightarrow \infty$ and using (2.7), we obtain

$$
\zeta\left(x_{s}\right)<\xi\left(x_{s}, \phi_{0}, \omega_{0}\right) \leq s=\zeta\left(x_{s}\right),
$$

which is a contradiction.

Hence, we proved that

$$
\lim _{n \rightarrow \infty} s_{n}=1
$$

Now, for any positive integer $q$, we have

$$
0 \preceq \phi_{n+q}-\phi_{n} \preceq \omega_{n}-\phi_{n} \preceq \omega_{n}-s_{n} \omega_{n}=\left(1-s_{n}\right) \omega_{n} \preceq\left(1-s_{n}\right) \omega_{0}
$$

and

$$
0 \preceq \omega_{n}-\omega_{n+q} \preceq \omega_{n}-\phi_{n} \preceq\left(1-s_{n}\right) \omega_{0} .
$$


Using (2.8) and Lemma 2.3 in [8], we deduce that $\left\{\phi_{n}\right\}$ and $\left\{\omega_{n}\right\}$ are Cauchy sequences. Then there exist $\phi^{*}, \omega^{*} \in \mathbb{V}$ such that $\left\{\phi_{n}\right\}$ converges to $\phi^{*}$ and $\left\{\omega_{n}\right\}$ converges to $\omega^{*}$. By (2.6), we have

$$
\phi_{0} \preceq \phi_{n} \preceq \phi^{*} \preceq \omega^{*} \preceq \omega_{n} \preceq \omega_{0}, \quad n=0,1,2, \cdots .
$$

Then we have

$$
o \preceq \omega^{*}-\phi^{*} \preceq \omega_{n}-\phi_{n} \preceq\left(1-s_{n}\right) \omega_{0} .
$$

Passing to the limit as $n \rightarrow \infty$, using (2.8) and Lemma 2.4 in [8], we obtain $\phi^{*}=\omega^{*}$. Set

$$
v^{*}=\phi^{*}=\omega^{*} .
$$

Then we have $v^{*} \in\left[\phi_{0}, \omega_{0}\right]$. Since $H$ is mixed monotone, using (2.9), we obtain

$$
\phi_{n+1}=H\left(\phi_{n}, \omega_{n}\right) \preceq H\left(v^{*}, v^{*}\right) \preceq H\left(\omega_{n}, \phi_{n}\right)=\omega_{n+1} .
$$

Passing to the limit as $n \rightarrow \infty$, we get

$$
v^{*} \preceq H\left(x^{*}, x^{*}\right) \preceq x^{*}
$$

which yields

$$
G F\left(v^{*}, v^{*}\right)=H\left(v^{*}, v^{*}\right)=v^{*} .
$$

Let us prove now that $v^{*}$ is the unique point in $Q_{\eta}$ satisfying the above equality. Suppose that $w^{*} \in Q_{\eta}$ is such that

$$
H\left(w^{*}, w^{*}\right)=w^{*} .
$$

Let

$$
\rho=\sup \left\{0<c \leq 1: c w^{*} \preceq v^{*} \preceq c^{-1} w^{*}\right\}
$$

Observe that $\rho \in(0,1]$ and

$$
\rho w^{*} \preceq v^{*} \preceq \rho^{-1} v^{*} .
$$

As in the proof of $s=1$, arguing by contradiction, we can prove that $\rho=1$. Then from (2.10), we have

$$
v^{*}=w^{*} .
$$

Thus we proved that $v^{*}$ is the unique solution in $Q_{\eta}$ to the operator equation 2.2 .

Now, we present some results that can be deduced from Theorem 2.1.

Let $\xi_{i}:(\alpha, \beta) \times Q \rightarrow(0, \infty), i=1,2$ be two functions with the following properties:

(P1) for all $i=1,2$, we have $\xi(x, v) \in(\zeta(x), 1)$ for all $(x, v) \in(\alpha, \beta) \times Q$;

(P2) for any $x \in(\alpha, \beta), \xi(x, u, v)=\min \left\{\xi_{1}(x, u), \xi_{2}(x, v)\right\}$ is increasing in $u$ for fixed $v$ and decreasing in $v$ for fixed $u$.

We denote by $\mathcal{T}$ the set of operators $T: Q \rightarrow Q$ such that

(T1) $T$ is increasing;

(T2) for all $(x, v) \in(\alpha, \beta) \times Q$, we have

$$
T(\zeta(x) v) \succeq \xi_{1}(x, v) T v .
$$

We denote by $\mathcal{S}$ the set of operators $S: Q \rightarrow Q$ such that

(S1) $S$ is decreasing;

(S2) for all $(x, v) \in(\alpha, \beta) \times Q$, we have

$$
S\left([\zeta(x)]^{-1} v\right) \succeq \xi_{2}(x, v) S v .
$$


Corollary 2.2. Let $(T, S, G) \in \mathcal{T} \times \mathcal{S} \times G$. Suppose that there exists $\eta \in Q, \eta \neq 0$ such that

$$
\frac{\zeta\left(x_{0}\right)}{\xi\left(x_{0}, \eta, \eta\right)} \eta \preceq G(T \eta+S \eta) \preceq \frac{1}{\zeta\left(x_{0}\right)} \eta
$$

for some $x_{0} \in(\alpha, \beta)$. Then the operator equation

$$
G(T v+S v)=v
$$

has a unique solution $v^{*} \in Q_{\eta}$.

Proof. Observe that the operator

$$
F(v, w)=T u+S w, \quad(v, w) \in Q \times Q
$$

belongs to the set $\mathcal{F}$. From Theorem 2.1, the operator equation

$$
G F(v, v)=v,
$$

which is equivalent to

$$
G(T v+S v)=v
$$

has a unique solution $v^{*} \in Q_{\eta}$.

Let $\xi_{1}:(\alpha, \beta) \times Q \rightarrow(0, \infty)$ and $\xi_{2}:(\alpha, \beta) \times Q \times Q \rightarrow(0, \infty)$ be two function satisfying the following assumptions:

(A1) $\xi_{1}(x, v), \xi_{2}(x, v, w) \in(\zeta(x), 1)$ for all $(x, v, w) \in(\alpha, \beta) \times Q \times Q$;

(A2) for any $x \in(\alpha, \beta), \xi(x, v, w)=\min \left\{\xi_{1}(x, v), \xi_{2}(x, v, w)\right\}$ is increasing in $v$ for fixed $w$ and decreasing in $w$ for fixed $v$.

We denote by $\mathcal{B}$ the set of operators $B: Q \rightarrow Q$ such that

(B1) $B$ is increasing;

(B2) $B(\zeta(x) v) \succeq \xi_{1}(x, v) B v, \quad(x, v) \in(\alpha, \beta) \times Q$.

We denote by $\mathcal{C}$ the set of operators $C: Q \times Q \rightarrow Q$ such that

(C1) $C$ is mixed monotone;

(C2) $C\left(\zeta(x) v,[\zeta(x)]^{-1} w\right) \succeq \xi_{2}(v, w) C(v, w), \quad(x, v, w) \in(\alpha, \beta) \times Q \times Q$.

Corollary 2.3. Let $(B, C, G) \in \mathcal{B} \times \mathcal{C} \times \mathcal{G}$. Suppose that there exists $\eta \in Q, \eta \neq 0$ such that

$$
\frac{\zeta\left(x_{0}\right)}{\xi\left(x_{0}, \eta, \eta\right)} \eta \preceq G(B \eta+C(\eta, \eta)) \preceq \frac{1}{\zeta\left(x_{0}\right)} \eta
$$

for some $x_{0} \in(\alpha, \beta)$.

Then the operator equation

$$
G(B v+C(v, v))=v
$$

has a unique solution in $Q_{\eta}$.

Proof. We observe that the operator

$$
F(v, w)=B v+C(v, w), \quad(v, w) \in Q \times Q
$$

belongs to the set $\mathcal{F}$. From Theorem 2.1, the operator equation

$$
G F(v, v)=v,
$$

which is equivalent to

$$
G(B v+C(v, v))=v
$$

has a unique solution in $Q_{\eta}$. 


\section{An application to integral equations}

Consider the integral equation

$$
\int_{0}^{x} \int_{0}^{1} \vartheta(\tau, y)[f(\tau, v(y))+g(\tau, v(y), v(y))] d y d \tau=v(x), \quad x \in[0,1],
$$

where $\vartheta:[0,1] \times[0,1] \rightarrow[0, \infty), f:[0,1] \times[0, \infty) \rightarrow[0, \infty)$ and $g:[0,1] \times[0, \infty) \times[0, \infty) \rightarrow[0, \infty)$ are regular functions.

Let $\left(\mathbb{V}, N, \mathbb{T}_{m}\right)$ be the PB-space, where $\mathbb{V}=C([0,1])$ is the set of real continuous functions in $[0,1]$ and $N: \mathbb{V} \rightarrow \mathbb{D}$ is given by

$$
N(u)(x)=\left\{\begin{array}{lll}
0 & \text { if } & x \leq 0 \\
\frac{x}{x+\max _{0 \leq z \leq 1}|u(z)|} & \text { if } & x>0
\end{array}, \quad u \in \mathbb{V} .\right.
$$

Here $\mathbb{T}_{m}$ is the $T$-norm given by $\mathbb{T}_{m}(\mu, \lambda)=\min \{\mu, \lambda\}$ for $\mu, \lambda \in[0,1]$. We consider the normal cone $Q$ given by

$$
Q=\{u \in \mathbb{V}: u(x) \geq 0, \text { for all } x \in[0,1]\} .
$$

Let $\xi:(0,1) \rightarrow(0,1)$ be a function such that

$$
\xi(x) \in(x, 1), \quad x \in(0,1) .
$$

Let $\mathbb{F}$ be the set of functions $f:[0,1] \times[0, \infty) \rightarrow[0, \infty)$ such that for a fixed $0 \leq s \leq 1$, the function $f(s, \cdot)$ is increasing in $[0,1]$ and

$$
f(s, x z) \geq \xi(x) f(s, z), \quad(x, z) \in(0,1) \times[0, \infty) .
$$

Let $\mathbb{G}$ be the set of functions $g:[0,1] \times[0, \infty) \times[0, \infty) \rightarrow[0, \infty)$ such that for a fixed $0 \leq s \leq 1$, the function $g(s, \cdot, \cdot)$ is mixed monotone and

$$
g\left(s, x z, x^{-1} w\right) \geq \xi(x) g(s, z, w), \quad(x, z, w) \in(0,1) \times[0, \infty) \times[0, \infty) .
$$

Theorem 3.1. Let $(f, g) \in \mathbb{F} \times \mathbb{G}$. Suppose that there exists $\eta \in Q, \eta \neq 0$ and $x_{0} \in(0,1)$ such that for all $x \in[0,1]$,

$$
\frac{x_{0}}{\xi\left(x_{0}\right)} \eta(x) \leq \int_{0}^{x} \int_{0}^{1} \vartheta(\tau, y)[f(\tau, \eta(y))+g(\tau, \eta(y), \eta(y))] d y d \tau \leq \frac{1}{x_{0}} \eta(x) .
$$

Then (3.1) has a unique solution $v^{*} \in Q_{\eta}$.

Proof. Observe that the operator $G: \mathbb{V} \rightarrow \mathbb{V}$ be the operator defined by

$$
(G v)(x)=\int_{0}^{x} v(y) d y, \quad x \in[0,1]
$$

belongs to the set $\mathcal{G}$. Let $B: Q \rightarrow Q$ be the operator given by

$$
(B v)(x)=\int_{0}^{1} \vartheta(x, y) f(x, v(y)) d y, \quad x \in[0,1] .
$$

It is not difficult to see that $B \in \mathcal{B}$ with $\zeta(x)=x, x \in(0,1)$ and $\xi_{1}(x, v)=\xi(x),(x, v) \in(0,1) \times Q$. Let $C: Q \times Q \rightarrow Q$ be the operator defined by

$$
C(v, w)(x)=\int_{0}^{1} \vartheta(x, y) g(x, v(y), w(y)) d y, \quad x \in[0,1] .
$$

Then $C \in \mathcal{C}$ with $\xi_{2}(x, v, w)=\xi(x),(x, v, w) \in(0,1) \times Q \times Q$. Moreover, from (3.2), there exists $\eta \in Q$, $\eta \neq 0$ such that

$$
\frac{\zeta\left(x_{0}\right)}{\xi\left(x_{0}\right)} \eta \preceq G(B \eta+C(\eta, \eta)) \preceq \frac{1}{\zeta\left(x_{0}\right)} \eta,
$$

for some $x_{0} \in(0,1)$. Using Corollary 2.3, we obtain that the operator equation

$$
G(B v+C(v, v)))=v,
$$

which is equivalent to the integral equation (3.1), has a unique solution in $Q_{\eta}$. 


\section{Acknowledgements}

This project was funded by the National Plan for Science, Technology and Innovation (MAARIFAH), King Abdulaziz City for Science and Technology, Kingdom of Saudi Arabia, Award Number (12-MAT 2913-02).

\section{References}

[1] H. Amann, Fixed point equations and nonlinear eigenvalue problems in ordered Banach spaces, SIAM Rev., 18 (1976), 620-709.1

[2] R. I. Avery, A. C. Peterson, Three positive fixed points of nonlinear operators on ordered Banach spaces, Comput. Math. Appl., 42 (2001), 313-322.1.

[3] I. Beg, A. Latif, R. Ali, A. Azam, Coupled fixed point of mixed monotone operators on probabilistic Banach spaces, Archivum Math., 37 (2001), 1-8.1

[4] A. Bharucha-Reid, Fixed point theorems in probabilistic analysis, Bull. Amer. Math. Soc., 82 (1976), 641-657.1

[5] S. S. Chang, Y. L. Cho, S. M. Kang, Probabilistic metric spaces and nonlinear operator theory, Nova Science Publishers, Inc., Huntington, New York, (2001). 1

[6] Y. J. Cho, Th. M. Rassias, R. Saadati, Stability of functional equations in random normed spaces, Springer, New York, (2013). 1

[7] D. Guo, V. Lakshmikantham, Nonlinear Problems in Abstract Cones, Academic Press, Boston, (1988).1]

[8] M. Jleli, B. Samet, Positive fixed points for convex and decreasing operators in probabilistic Banach spaces with an application to a two-point boundary value problem, Fixed Point Theory Appl., 2015 (2015), 19 pages.1, 2, 2

[9] M. A. Krasnosel'skii, Positive solutions of operators equations, Noordoff, Groningen, (1964). 1 ,

[10] K. Menger, Statistical metrics, Proc. Nat. Acad. Sci. USA., 28 (1942), 535-537.1]

[11] B. Schweizer, A. Sklar, Probabilistic metric spaces, North-Holland Publiahing Co., New York, (1983). 1

[12] C. B. Zhai, D. R. Anderson, A sum operator equation and applications to nonlinear elastic beam equations and Lane-Emden-Fowler equations, J. Math. Anal. Appl., 375 (2011), 388-400.1

[13] C. B. Zhai, X. M. Cao, Fixed point theorems for $\tau-\varphi$-concave operators and applications, Comput. Math. Appl., 59 (2010), 532-538.1

[14] C. B. Zhai, W. P. Yan, C. Yang, A sum operator method for the existence and uniqueness of positive solutions to Riemann-Liouville fractional differential equation boundary value problems, Commun. Nonlinear Sci. Numer. Simul., 18 (2013), 858-866.1

[15] C. B. Zhai, L. L. Zhang, New fixed point theorems for mixed monotone operators and local existence-uniqueness of positive solutions for nonlinear boundary value problems, J. Math. Anal. Appl., 382 (2011), 594-614.1.

[16] Z. Zhao, X. Du, Fixed points of generalized e-concave (generalized e-convex) operators and their applications, J. Math. Anal. Appl., 334 (2007), 1426-1438.1 